\title{
Associations between body mass index and molecular subtypes as well as other clinical characteristics of breast cancer in Chinese women
}

This article was published in the following Dove Press journal:

Therapeutics and Clinical Risk Management

25 March 2013

Number of times this article has been viewed

Fei-Yu Chen

Hui-Ying Ou

Shou-Man Wang

Yu-Hui Wu

Guo-Jiao Yan

Li-Li Tang

Department of Breast Surgery, Xiangya Hospital, Central South University, Changsha City, Hunan Province, People's Republic of China

Correspondence: Li-Li Tang

Department of Breast Surgery, Xiangya Hospital, Central South University, No 87 Xiangya Road, Changsha City, Hunan Province, 410008 , People's Republic of China Tel/Fax +86 37I 89753516

Email tang_breast@yahoo.cn
Background: Several studies have shown a positive association between body mass index (BMI) and the development of hormone receptor-positive breast cancer in postmenopausal women; however, the associations between BMI groups and molecular subtypes have yet to be well defined in premenopausal breast cancer patients.

Methods: A total of 2465 female breast cancer patients diagnosed at our institution were recruited for this study. Clinicopathologic information (including age, body height and weight, as well as tumor subtypes and stages) was collected; analyses of these characteristics and the associations between them were performed.

Results: A total of 1951 cases were included in the study. The mean age was 47.3 years, the majority of patients were of normal weight, premenopausal, had stage 2 cancer, and did not present with positive nodes. The prevalence of the luminal A, luminal $\mathrm{B}$, human epidermal growth factor receptor $2+$, and triple-negative subtypes were $57.8 \%, 11.6 \%, 6.1 \%$, and $24.5 \%$, respectively. There were significant differences in the clinicopathologic features among BMI groups in premenopausal patients. The case-only odds ratio (OR) analysis revealed that normal weight patients tended to have luminal $\mathrm{B}$ cancer $(\mathrm{OR}=1.4, P=0.206)$, and overweight and obese patients tended to have triple-negative cancer in premenopausal patients $(\mathrm{OR}=2.8, \mathrm{OR}=3.7$, respectively; $P<0.001$ ).

Conclusion: In Chinese women, breast cancer came with these characteristics: young mean age (premenopause), luminal A subtype, and the majority of them were within a normal weight range. In premenopausal patients, underweight patients tended to have luminal A, lower human epidermal growth factor receptor $2+$ expression, stage 1 and no positive node cancer. However, overweight and obese patients tended to have a triple-negative, stage 3, and lymph node metastatic cancer.

Keywords: breast cancer, body mass index, molecular subtype, Chinese

\section{Introduction}

Heterogeneity is the most characteristic feature of breast cancer, which is seen in many aspects of the patients from clinical features to pathological and racial/ethnic properties; ${ }^{1}$ this makes the identification of risk factors for breast cancer more difficult. Many studies in Western countries have shown that high body mass index (BMI) is an important risk factor for breast cancer among postmenopausal women. While postmenopausal women show a positive association between high BMI and breast cancer risk, a negative association is found in premenopausal women. ${ }^{2-4}$ However, in Asian populations, a recent study from India indicated that increased BMI, large waist size, and large hip size were risk factors for breast cancer both in premenopausal and postmenopausal women. ${ }^{5}$ On the other hand, research from the People's Republic of 
China found that BMI at diagnosis was positively correlated with the risk of breast cancer in postmenopausal women, but it was not related to the risk in premenopausal women. ${ }^{6}$

Although the relevance of BMI as a prognostic factor in breast cancer remains controversial, the majority of the breast cancer studies to date have suggested that higher BMI at diagnosis is associated with a poorer prognosis. ${ }^{7-12}$ A study from Korea indicated that individuals who are underweight (UW) should also be considered to be at high risk for death and recurrence after breast cancer surgery. ${ }^{13}$ Several studies have shown that in postmenopausal women, BMI is highly associated with the development of hormone receptor-positive breast cancer rather than hormone receptornegative breast cancer; ${ }^{14-17}$ However, few studies have taken BMI together with tumor subtypes, tumor stage, and nodal involvement for revealing possible relationships between them, and the majority of studies are conducted in Western countries. ${ }^{14,18,19}$

In the present study, using a database of 2465 breast cancer patients, we selected clinical features (especially BMI and tumor subtypes) to find any potential relationships between them, especially to determine the numbers and distribution of these features within the BMI groups in both premenopausal and postmenopausal patients in the Chinese population. An odds ratio (OR) analysis was also carried out to show the strength of BMI as a risk factor for different molecular subtypes of breast cancer.

\section{Materials and methods Clinical data}

We established a database of all female patients $(n=2465)$ diagnosed with breast cancer and who underwent surgical treatment between December 2001 and November 2011 at the Department of Breast Surgery, Xiangya Hospital of Central South University. General clinicopathologic information (such as age, body height, weight, tumor stage, and state of lymph nodes) was collected from the database (height and weight were measured in the hospital prior to surgery).

The expression levels of ER (estrogen receptor), PR (progesterone receptor), Ki-67 (cell proliferation marker), and human epidermal growth factor receptor 2 (HER2) in the cancer tissue samples were examined using immunohistochemistry by experienced pathologists. ER and PR expression were considered to be positive if more than $1 \%$ of cells showed positive staining of nuclei in a single section. The intensity and the pattern of HER2 staining in the membrane of tumor cells were evaluated using a score system for the categorization of the tumors. In brief, scores of 0 and
$1+$ (weak immunostaining in less than $30 \%$ of tumor cells) were defined as HER2-negative; score 2+ (complete, strong membranous staining, in at least $10 \%$ but in less than $30 \%$ of tumor cells) was defined as equivocal; score 3+ (uniform intense membranous staining in above $30 \%$ of tumor cells) was defined as HER2-positive. Tumors that were scored as $2+$ were further assessed by fluorescence in situ hybridization (FISH). If FISH showed positive staining, these 2+ tumors were designated as HER2-positive as well. ${ }^{20,21}$ The study has been approved by the local ethics committee of medical research involving human subjects at Central South University.

Patients with tumors with a score of $2+$, but without the further assessment by FISH were excluded; in addition, there were some missing data - a total of 1951 (79.1\%) cases were included in this study. Based on the body weight index, where:

$$
\mathrm{BMI}=\text { weight }(\mathrm{kg}) / \text { height squared }(\mathrm{m})^{2}
$$

the patients were grouped into four categories following the World Health Organization guideline: ${ }^{22} \mathrm{UW}$ $\left(\mathrm{BMI}<18.5 \mathrm{~kg} / \mathrm{m}^{2}\right)$; normal weight $(\mathrm{NW}),(\mathrm{BMI}=18.5-$ $\left.24.99 \mathrm{~kg} / \mathrm{m}^{2}\right)$; overweight (OW), $\left(\mathrm{BMI}=25.0-29.99 \mathrm{~kg} / \mathrm{m}^{2}\right)$; and obese $(\mathrm{OB}),\left(\mathrm{BMI} \geq 30.0 \mathrm{~kg} / \mathrm{m}^{2}\right)$. Using the newly recommended method for categorizing breast cancer subtypes, ${ }^{23}$ the tumors were grouped into four subtypes: (1) luminal A-, ER-, and/or PR-positive, HER2-negative (HER2-), and Ki-67 low(<14\%); (2) luminal B-, ER-, and/or PR-positive, HER2-, and Ki-67 high ( $\geq 14 \%$ ), or ER- and/or PR-positive, any Ki-67, and HER2-positive (HER2+); (3) HER2+, HER2overexpressed or amplified, and ER- and PR-absent; and (4) triple-negative, ER- and PR-absent, and HER2-.

The code of tumor, lymph node, and metastasis (TNM) staging was in compliance with the National Comprehensive Cancer Network clinical practice guidelines for breast cancer 2011. The patients were categorized as premenopausal if they were younger than 51 years old and as postmenopausal if over 51 when they were diagnosed with the disease.

\section{Statistics}

The Chi-square test, Fisher's exact test, or analysis of variance were used to determine differences in the clinicopathologic features between BMI groups as appropriate. The case-only OR was used to evaluate the relative strength of association between BMI and a given tumor molecular subtype (ie, luminal B, HER2+, or triple-negative) versus luminal A, which is the most common subtype. All statistical 
analyses were performed using the Statistical Package for the Social Sciences (SPSS version 17.0; IBM Corporation, Armonk, NY, USA) for Windows (Microsoft Corporation, Redmond, WA, USA), and all tests of statistical significance were two-sided.

\section{Results}

Of the 1951 breast cancers in our databases, one datum was lost; 1127 (57.8\%) were luminal A, 227 (11.6\%) were luminal B, 119 (6.1\%) were HER2+, and 477 (24.5\%) were triple-negative.

There were only 1789 cases with TNM data, and the majority of the cases were stage 2 , which accounted for $61.0 \%$, and the least was stage 4 , which accounted for $0.8 \%$ $(15 / 1789)$. Therefore, in the following analysis that involved TNM staging, stage 4 was excluded based on the fact that there were too few cases. After surgery, we determined the state of the lymph nodes of our patients, and the data of 30 cases were lost. In the end, 1067 (55.5\%) cases had no positive nodes, $447(23.3 \%)$ cases had one to three positive nodes, and 407 $(21.2 \%)$ cases had more than three positive nodes.

The investigation also revealed a difference in BMI between premenopausal and postmenopausal breast cancer patients. Our data showed that the BMI of postmenopausal patients was significantly higher than that of premenopausal patients $\left(23.81 \pm 3.27 \mathrm{~kg} / \mathrm{m}^{2}\right.$ versus $\left.22.93 \pm 2.92 \mathrm{~kg} / \mathrm{m}^{2}, P<0.001\right)$.
Next, we examined the associations between BMI, age, molecular subtypes, TNM staging, and number of positive nodes. The mean age among BMI groups was significantly different $(P<0.001)$ : the OB group was the oldest $(54.1 \pm 12.3$ years $)$, and the UW group was the youngest $(45.4 \pm 12.9$ years). Table 1 shows that the clinicopathologic features of the patients are significantly different across the different BMI groups. Firstly, the molecular subtypes were quite different in the premenopausal BMI groups $(P=0.000)$; the UW and NW groups have more patients in luminal $\mathrm{A}$ and luminal $\mathrm{B}$, and have less patients in HER2+ and triple-negative. However, the OW and OB groups have more patients in triple-negative and have fewer patients in luminal A and luminal B. Despite this, there were no significant differences in molecular subtypes in postmenopausal BMI groups $(P=0.188)$. When we compared the distributions of molecular subtypes between premenopausal and postmenopausal patients, we found a significant difference. Luminal A was comparatively higher in premenopausal than in postmenopausal patients $(60.9 \%$ versus $51.9 \%)$, and the triple-negative reflected a reversal of this trend (23.3\% versus $26.7 \%)$. Secondly, we found a significant difference of tumor staging in pre- or postmenopausal BMI groups $(P=0.000, P=0.000)$. The UW and NW groups had more patients in stage 1 and fewer patients in stage 3 than the OW and OB groups in

Table I The distributions of the special clinicopathologic features among patients according to BMI group

\begin{tabular}{|c|c|c|c|c|c|c|c|c|c|c|}
\hline \multirow{2}{*}{$\begin{array}{l}\text { Characteristic } \\
\text { status }\end{array}$} & \multicolumn{5}{|c|}{ Premenopausal patients } & \multicolumn{5}{|c|}{ Postmenopausal patients } \\
\hline & $\begin{array}{l}\text { UW } \\
\text { n (\%) }\end{array}$ & $\begin{array}{l}\text { NW } \\
\text { n (\%) }\end{array}$ & $\begin{array}{l}\text { OW } \\
\text { n (\%) }\end{array}$ & $\begin{array}{l}\text { OB } \\
\text { n (\%) }\end{array}$ & $\begin{array}{l}\text { Subtotal } \\
\text { n (\%) }\end{array}$ & $\begin{array}{l}\text { UW } \\
\text { n (\%) }\end{array}$ & $\begin{array}{l}\text { NW } \\
\text { n (\%) }\end{array}$ & $\begin{array}{l}\text { OW } \\
\text { n (\%) }\end{array}$ & $\begin{array}{l}\text { OB } \\
\text { n (\%) }\end{array}$ & $\begin{array}{l}\text { Subtotal } \\
\text { n (\%) }\end{array}$ \\
\hline \multicolumn{11}{|c|}{ Molecular subtypes } \\
\hline Luminal A & $42(73.7)$ & $578(62.2)$ & I $48(54.8)$ & $10(50.0)$ & $778(60.9)$ & $12(46.2)$ & $227(5 \mid .7)$ & $88(50.3)$ & $22(66.7)$ & $349(51.9)$ \\
\hline Luminal B & $6(10.5)$ & $118(12.7)$ & $18(6.7)$ & I (5.0) & $143(|| .2)$ & $3(11.5)$ & $62(14.1)$ & I5 (8.6) & $4(12.1)$ & $84(12.5)$ \\
\hline HER2+ & $0(0)$ & $44(4.7)$ & $14(5.2)$ & I (5.0) & $59(4.6)$ & I (3.8) & $44(10.0)$ & $13(7.4)$ & $2(6.1)$ & $60(8.9)$ \\
\hline Triple-negative & $9(15.8)$ & $190(20.4)$ & $90(33.3)$ & $8(40.0)$ & $297(23.3)$ & $10(38.5)$ & $106(24.2)$ & $59(33.7)$ & $5(I 5.2)$ & $180(26.7)$ \\
\hline$P$-value & $0.000 I^{*}$ & & & & & 0.118 & & & & $0.00005^{* *}$ \\
\hline \multicolumn{11}{|l|}{ TNM staging } \\
\hline I & $12(24.0)$ & $127(14.3)$ & $24(9.2)$ & I (4.8) & $164(13.4)$ & $4(16.0)$ & $55(16.8)$ & $15(9.1)$ & $3(9.4)$ & $77(\mid 4.0)$ \\
\hline 2 & $26(52.0)$ & $54 \mid(60.7)$ & $136(51.9)$ & $12(57.1)$ & $715(58.4)$ & $18(72.0)$ & $249(75.9)$ & $87(52.7)$ & $22(68.8)$ & $376(68.4)$ \\
\hline 3 & $12(24.0)$ & $223(25.0)$ & $102(38.9)$ & $8(38.1)$ & $345(28.2)$ & $3(12.0)$ & $24(7.3)$ & $63(38.2)$ & $7(21.9)$ & $97(17.6)$ \\
\hline$P$-value & $0.000 I^{*}$ & & & & & $6 \times 10^{-14} *$ & & & & $0.000009 * *$ \\
\hline \multicolumn{11}{|c|}{ Number of positive nodes } \\
\hline 0 & $33(6 \mathrm{I} .1)$ & $526(57.3)$ & $136(50.6)$ & $8(40.0)$ & $703(55.8)$ & $19(73.1)$ & $246(56.9)$ & $83(48.8)$ & $16(50.0)$ & $364(55.2)$ \\
\hline $1-3$ & $8(14.8)$ & $222(24.2)$ & $60(22.3)$ & $9(45.0)$ & $299(23.7)$ & $5(19.2)$ & $90(20.8)$ & $43(25.3)$ & $10(31.3)$ & I 48 (22.4) \\
\hline$>3$ & $13(24.1)$ & $170(18.5)$ & $73(27.1)$ & $3(I 5.0)$ & $259(20.5)$ & $2(7.7)$ & $96(22.2)$ & $44(25.9)$ & $6(18.8)$ & I 48 (22.4) \\
\hline$P$-value & $0.009 *$ & & & & & 0.167 & & & & 0.588 \\
\hline
\end{tabular}

Notes: $* P$-values were obtained by comparing the four BMl groups using the Chi-square test for differences in frequencies of clinicopathologic features; $P<0.05$ was considered to be statistically significant; **P-values were obtained by comparing the pre- and postmenopausal patients using the Chi-square test for differences in frequencies of clinicopathologic features; $P<0.05$ was considered to be statistically significant.

Abbreviations: BMI, body mass index; UW, underweight; n, number; NW, normal weight; OW, overweight; OB, obese; HER2+, human epidermal growth factor receptor 2-positive; TNM, tumor, lymph node, and metastasis. 
both pre- and postmenopausal patients. There was also a significant difference in distributions of TNM staging between pre- and postmenopausal patients $(P=0.000)$; a larger percentage of premenopausal patients have stage 3 cancer when compared to postmenopausal patients. Finally, the status of lymph nodes was significantly different in the premenopausal BMI groups $(P=0.009)$; the UW and NW groups had more patients with no positive nodes. Meanwhile, the OB group had more patients with one to three positive nodes, but the status of lymph nodes was not significantly different in the postmenopausal BMI groups $(P=0.167)$. Furthermore, there was also no significant variation in terms of the status of lymph nodes between pre- and postmenopausal patients $(P=0.588)$.

Case-only analysis using disease subtypes is a useful exploratory tool to uncover etiologic heterogeneity. ${ }^{24}$ In our study, the case-only OR estimates the relative strength of the association between BMI and a given disease subtype (luminal B, HER2+, or triple-negative) versus luminal A, which is the most common subtype, acting as the comparison group. As shown in Table 2, when compared to luminal A, luminal $\mathrm{B}$ tended to be more dominant in the NW premenopausal patients $(\mathrm{OR}=1.4,95 \%$ confidence interval $[\mathrm{CI}]=0.6-3.4$, $P=0.206$ ), although this finding was not significant. Triplenegative tended to be found among the OW and OB premenopausal patients $(\mathrm{OR}=2.8,95 \% \mathrm{CI}=1.3-6.1 ; \mathrm{OR}=3.7$, $95 \% \mathrm{CI}=1.2-12.1$, respectively; $P<0.001)$. We did not find significant differences between luminal A and HER2+ in the different BMI groups. Furthermore, there was no significant difference between molecular subtypes in the BMI groups in postmenopausal patients.

\section{Discussion}

In this study, we showed that among Chinese women, the BMI of the majority of breast cancer patients was NW, accounting for $70.2 \%$ of total patients, which is higher than the patients in Western countries; only $2.7 \%$ of breast cancer patients had a BMI classifying them as OB, which is lower than in Western countries. ${ }^{12,18,25,26}$ A young diagnostic age (average age of 47.3 years, $65.5 \%$ of which were premenopausal patients) and different diet in Chinese women may contribute to this difference. Likewise, BMI in postmenopausal patients was significantly higher than that in premenopausal patients $(P<0.001)$.

The age at diagnosis of breast cancer in our patients ranged from 21 years to 88 years, and the mean age was 47.3 years, which is younger than the mean age in both Hong Kong, which was 55.4 years, and Norway, which was 57.5 years. ${ }^{6,25}$ Patient and tumor characteristics are presented in Table 1. In our patients, the majority of patients' BMI fell in the range of NW, which accounted for $70.2 \%$; the next largest group was comprised of those in the OW range (22.8\%), and the smallest was the OB group (2.7\%), which was contrary to what was observed in Western countries..$^{12,18,25,26}$ Of our patients, $65.5 \%$ of the women in the premenopausal group were younger than 51 years, and $34.5 \%$ in the postmenopausal group were 51 years or older, which was also quite different from what was observed in the USA, where the majority of patients were postmenopausal. ${ }^{27}$

There are many studies subclassifying triple-negative tumors as basal-like or unclassified, based on epidermal growth factor receptor and cytokeratin 5/6 expression; ${ }^{28,29}$ however, since the data for epidermal growth factor receptor

Table 2 Case-only ORs comparing luminal B, HER2+, and triple-negative with luminal A breast cancer

\begin{tabular}{|c|c|c|c|c|c|c|c|c|}
\hline \multirow[t]{3}{*}{ Characteristic } & \multirow[t]{3}{*}{ Status } & \multicolumn{7}{|c|}{ Molecular subtypes } \\
\hline & & \multirow{2}{*}{$\begin{array}{l}\text { Luminal A } \\
\mathrm{n}\end{array}$} & \multicolumn{2}{|c|}{ Luminal B } & \multicolumn{2}{|c|}{ HER2+ } & \multicolumn{2}{|c|}{ Triple-negative } \\
\hline & & & $\mathbf{n}$ & OR $(95 \% \mathrm{Cl})$ & $\mathbf{n}$ & OR $(95 \% \mathrm{Cl})$ & $\mathbf{n}$ & OR $(95 \% \mathrm{CI})$ \\
\hline \multicolumn{9}{|l|}{ BMI } \\
\hline \multirow[t]{5}{*}{ Premenopausal } & UW & 42 & 6 & I.0 (referent) & 0 & I.0 (referent) & 9 & I.0 (referent) \\
\hline & NW & 577 & 118 & I.4 (0.6-3.4) & 44 & & 190 & $1.5(0.7-3.2)$ \\
\hline & ow & 148 & 18 & $0.9(0.3-2.3)$ & 14 & & 90 & $2.8(1.3-6.1)^{\#}$ \\
\hline & $\mathrm{OB}$ & 10 & I & $0.7(0.1-6.5)$ & I & & 8 & $3.7(1.2-12.1)^{\#}$ \\
\hline & $P$-value & & & 0.206 & & & & $0.000 I *$ \\
\hline \multirow[t]{5}{*}{ Postmenopausal } & UW & 12 & 3 & I.0 (referent) & I & I.0 (referent) & 10 & I.0 (referent) \\
\hline & NW & 228 & 62 & I.I (0.3-4.0) & 44 & $2.3(0.3-18.3)$ & 106 & $0.6(0.2-1.3)$ \\
\hline & OW & 88 & 15 & $0.7(0.2-2.7)$ & 13 & I.8 (0.2-14.8) & 59 & $0.8(0.2-2.0)$ \\
\hline & $\mathrm{OB}$ & 22 & 4 & $0.7(0.1-3.8)$ & 2 & $1.0(0.1-13.3)$ & 5 & $0.3(0.1-1.0)$ \\
\hline & $P$-value & & & 0.468 & & 0.566 & & 0.060 \\
\hline
\end{tabular}

Notes: ${ }^{*} P$-values were obtained from comparisons of each molecular subtype to luminal $A$ using the Chi-square test; $P<0.05$ was considered to be statistically significant; "compared with luminal A, triple-negative tends to be prevalent in OW and OB premenopausal patients.

Abbreviations: OR, odds ratio; HER2+, human epidermal growth factor receptor 2-positive; n, number; $\mathrm{Cl}$, confidence interval; BMI, body mass index; UW, underweight; NW, normal weight; OW, overweight; OB, obese. 
and cytokeratin 5/6 were not available, we grouped all the negative tumors into the triple-negative tumors. Our study showed that luminal A ( $57.8 \%$ of total patients) was the most prevalent subtype, which is similar to the finding observed in studies of Chinese patients (48.6\% and 50.0\%) and Polish patients $(69 \%))^{29-31}$ The luminal B subtype was present in $11.6 \%$ of the patients, which is lower in comparison with other Chinese reports (16.7\% and $15.1 \%$, respectively), ${ }^{30,31}$ but still higher than what a Polish study has reported $(6 \%){ }^{29}$ The HER2+ subtype accounted for $6.1 \%$ in our study, which is lower than other reports $(13.7 \%, 10.87 \%$, and $8 \%) .{ }^{29-31}$ In addition, the triple-negative subtype accounted for $24.5 \%$ of patients, which is similar to the finding in the northeast Chinese population $(23.08 \%),{ }^{31}$ but this finding is higher than that in Shanghai (12.9\%) and Poland (18\%). ${ }^{29,30}$ The following reasons may explain these differences: firstly, the prevalence of breast cancer subtypes appears to differ among different races or ethnicities. ${ }^{1}$ Secondly, we took the level of Ki-67 as a marker for classifying luminal A and part of luminal B for the first time, therefore some of the luminal A subtype would have fallen into luminal B. Lastly, some patients with tumors that showed 2+ HER2/neu immunohistochemistry staining were not assessed by FISH, and were thus excluded from our study. From our study, $61.0 \%$ cases were with stage 2 and $24.7 \%$ were with stage 3 breast cancer, and only $13.5 \%$ cases were in stage 1. This is quite different from the US and Norway reports (stage 2, 50.2\% and $41.3 \%$; stage 3, 3.1\% and $4.6 \%$; stage $1,46.7 \%$ and $48.8 \%$, respectively). ${ }^{25,27}$ Moreover, the metastasis to lymph nodes in our patients was worse than in the US report; $44.5 \%$ versus $36.8 \%$ cases had positive nodes, $23.3 \%$ versus $26.3 \%$ cases had one to three positive nodes, and $21.2 \%$ versus $10.5 \%$ cases had more than three positive nodes, respectively. ${ }^{27}$ These data showed that Chinese patients were diagnosed with breast cancer relatively later than the US patients, indicating room for further improvement in the early diagnosis of breast cancer in the People's Republic of China.

As we have shown in Chinese patients, the older individuals tended to have a higher BMI. The patient's hormone status would contribute to this BMI variance, and the hormone status would also play a role in the pathogenesis of some special subtypes of breast cancer.

The profile of the molecular subtypes in the BMI groups showed significant difference in premenopausal patients, but there was no significant difference in postmenopausal patients. In premenopausal patients, the distribution of molecular subtypes was as follows: luminal A was higher in the UW group, luminal B was higher in the NW group,
HER2+ was lower in the UW group, and triple-negative was higher in the OW and OB groups. Similarly, when we used case-only ORs comparing each subtype to luminal A, we found that triple-negative tended to be found in $\mathrm{OW}$ and $\mathrm{OB}$ premenopausal patients $(\mathrm{OR}=2.8$ and $\mathrm{OR}=3.7$, respectively; $P<0.001$ ), and luminal $\mathrm{B}$ tended to be found in NW, although there was no significant difference. We did not find any previous studies about an association between molecular subtypes and BMI groups in the premenopausal breast cancer patients.

Although there was no significant difference in the molecular subtypes of the BMI groups among postmenopausal patients, luminal A tended to be higher in the OB group than in the other groups. This association is likely attributable to the positive relationship between BMI and endogenous estrogen levels since adipose tissue is the primary source of estrogen in postmenopausal women. It should be mentioned that a study of postmenopausal patients by Suzuki et al ${ }^{14}$ found that there was positive association between obesity and the development of ER+ PR+ tumors in never-users of postmenopausal hormones (relative risk, $R R=1.90$ ). In addition, Phipps et $\mathrm{al}^{17}$ found that among postmenopausal women not currently using menopausal hormone therapy, BMI was associated with the risk of luminal tumors (OR comparing the highest versus the lowest quartiles $=1.7$ ) and suggested that it was associated with the risk of triple-negative tumors $(\mathrm{OR}=2.7)$. However, in our study, the triple-negative was relatively higher in the UW and OW groups than in the other groups in postmenopausal patients. It may be that the prevalence of breast cancer subtypes and the distribution of BMI appear to differ among different races or ethnicities. ${ }^{1}$

In our study, we found that stage 1 was higher in the UW group and stage 3 was higher in the OW and OB groups, either in pre- or postmenopausal patients, and these findings were significant. When comparing the node state between BMI groups, we found that there was significant difference in premenopausal patients. Moreover, the status of "no-positive" node was higher in the UW group; one node patients (one to three positive nodes) were seen more in the OB group, and multinode cases (more than three positive nodes) were higher in the OW group. Although there was no significant difference among postmenopausal patients, the trend of node state between BMI groups was similar to that of premenopausal patients.

From our study, the UW group tended to be luminal A, stage 1, had lower HER2 expression, and had no positive nodes, suggesting a prospective outcome, ${ }^{32}$ especially in premenopausal patients. In addition, the OW and OB groups 
tended to be triple-negative, stage 3 , and presented with lymph node metastasis, which suggested a poorer prognosis, ${ }^{30}$ which was also especially observed in premenopausal patients. However, we did not get overall follow-up data, so we could not analyze the prognosis between BMI groups. From the different populations, the BMI-associated prognosis was quite different in breast cancer patients. A study from the US showed that being OB was more likely to be associated with increased risk of recurrence and poorer survival. ${ }^{27}$ The findings from the study from Norway were similar to those of the US, and showed that those with a $\mathrm{BMI} \geq 30 \mathrm{~kg} / \mathrm{m}^{2}$ had a 1.47 times higher risk of dying during follow-up than women with a BMI of $18.5-25 \mathrm{~kg} / \mathrm{m}^{2} .^{25}$ However, a study from Korea showed a significantly lower overall survival and breast cancer-specific survival in UW patients compared with the survival of NW patients after adjusting for known prognostic factors, which was not observed in OB patients; ${ }^{13}$ therefore, we need to follow up with our patients in order to obtain more conclusive information about the Chinese population.

There were some potential limitations in our study. Firstly, a total of $514(20.9 \%)$ patients were excluded because the tumors showing 2+ HER2/neu immunohistochemistry staining were not assessed by FISH, and some clinicopathologic data were missing. Secondly, we did not set a healthy population as a control. Thirdly, most of our patients came from the Hunan Province, which is in the center of the People's Republic of China, and this may not have been completely representational of the entire Chinese ethnicity. Thus, when comparing results, considerations should be made pertaining to the aforementioned limitations. In the future, epidemiological and biological evidence should be considered as well, in order to evaluate the differences across different studies; in addition, we also have to study the links between these differences, and their underlying epidemiological/biological background factors.

\section{Conclusion}

In Chinese women, breast cancer has the following characteristics: luminal A is the most common subtype; the majority of patients are NW; and the mean age of the patients is younger than in Western patients. In premenopausal patients, the UW group tended to be luminal A, stage 1, with lower HER2 expression, and exhibited no positive nodes; conversely, the OW and OB groups tended to be triple-negative, stage 3 , and presented with lymph node metastasis.

\section{Acknowledgments}

This study was supported by grant 2010-TP4053 from the China Hunan Provincial Science and Technology Department. The authors thank all of the professors, doctors, and nurses in the Breast Surgery Department of Xiangya hospital for collecting patient information, and the authors also wish to thank all of the professors, doctors, and technicians in the Pathology Department of Xiangya hospital. The authors also thank Professor Gou-Qiang Xiong from the School of Public Health, Central South University. All authors participated in the design and performance of the study; all authors wrote the manuscript together and approved the final submission; LT supported the study from his funding. The study has been approved by a local ethics committee of medical research involving human subjects at Xiangya Hospital, Central South University.

\section{Disclosure}

The authors report no conflicts of interest in this work.

\section{References}

1. Chlebowski RT, Chen Z, Anderson GL, et al. Ethnicity and breast cancer: factors influencing differences in incidence and outcome. J Natl Cancer Inst. 2005;97(6):439-448.

2. Tehard B, Clavel-Chapelon F. Several anthropometric measurements and breast cancer risk: results of the E3N cohort study. Int $J$ Obes (Lond). 2006;30(1):156-163.

3. van den Brandt PA, Spiegelman D, Yaun SS, et al. Pooled analysis of prospective cohort studies on height, weight, and breast cancer risk. Am J Epidemiol. 2000;152(6):514-527.

4. Morimoto LM, White E, Chen Z, et al. Obesity, body size, and risk of postmenopausal breast cancer: the Women's Health Initiative (United States). Cancer Causes Control. 2002;13(8):741-751.

5. Mathew A, Gajalakshmi V, Rajan B, et al. Anthropometric factors and breast cancer risk among urban and rural women in South India: a multicentric case-control study. Br J Cancer. 2008;99(1): 207-213.

6. Chow LW, Lui KL, Chan JC, et al. Association between body mass index and risk of formation of breast cancer in Chinese women. Asian J Surg. 2005;28(3):179-184.

7. Berclaz G, Li S, Price KN, et al; for International Breast Cancer Study Group. Body mass index as a prognostic feature in operable breast cancer: the International Breast Cancer Study Group experience. Ann Oncol. 2004;15(6):875-884.

8. Whiteman MK, Hillis SD, Curtis KM, McDonald JA, Wingo PA, Marchbanks PA. Body mass and mortality after breast cancer diagnosis. Cancer Epidemiol Biomarkers Prev. 2005;14(8):2009-2014.

9. Nichols HB, Trentham-Dietz A, Egan KM, et al. Body mass index before and after breast cancer diagnosis: associations with all-cause, breast cancer, and cardiovascular disease mortality. Cancer Epidemiol Biomarkers Prev. 2009;18(5):1403-1409.

10. Cleveland RJ, Eng SM, Abrahamson PE, et al. Weight gain prior to diagnosis and survival from breast cancer. Cancer Epidemiol Biomarkers Prev. 2007;16(9):1803-1811.

11. Loi S, Milne RL, Friedlander ML, et al. Obesity and outcomes in premenopausal and postmenopausal breast cancer. Cancer Epidemiol Biomarkers Prev. 2005;14(7):1686-1691. 
12. Demirkan B, Alacacioglu A, Yilmaz U. Relation of body mass index (BMI) to disease free (DFS) and distant disease free survivals (DDFS) among Turkish women with operable breast carcinoma. Jpn J Clin Oncol. 2007;37(4):256-265.

13. Moon HG, Han W, Noh DY. Underweight and breast cancer recurrence and death: a report from the Korean Breast Cancer Society. J Clin Oncol. 2009;27(35):5899-5905.

14. Suzuki R, Rylander-Rudqvist T, Ye W, Saji S, Wolk A. Body weight and postmenopausal breast cancer risk defined by estrogen and progesterone receptor status among Swedish women: A prospective cohort study. Int J Cancer. 2006;119(7):1683-1689.

15. Ahn J, Schatzkin A, Lacey JV Jr, et al. Adiposity, adult weight change, and postmenopausal breast cancer risk. Arch Intern Med. 2007;167(19): 2091-2102.

16. Enger SM, Ross RK, Paganini-Hill A, Carpenter CL, Bernstein L. Body size, physical activity, and breast cancer hormone receptor status: results from two case-control studies. Cancer Epidemiol Biomarkers Prev. 2000;9(7):681-687.

17. Phipps AI, Malone KE, Porter PL, Daling JR, Li CI. Body size and risk of luminal, HER2-overexpressing, and triple-negative breast cancer in postmenopausal women. Cancer Epidemiol Biomarkers Prev. 2008;17(8):2078-2086.

18. Stead LA, Lash TL, Sobieraj JE, et al. Triple-negative breast cancers are increased in black women regardless of age or body mass index. Breast Cancer Res. 2009;11(2):R18.

19. Millikan RC, Newman B, Tse CK, et al. Epidemiology of basal-like breast cancer. Breast Cancer Res Treat. 2008;109(1):123-139.

20. Carlson RW, Moench SJ, Hammond ME, et al; for NNCN HER2 Testing in Breast Cancer Task Force. HER2 testing in breast cancer: NCCN Task Force report and recommendations. J Natl Compr Canc Netw. 2006;4 Suppl 3:S1-S22; quiz S23-S24.

21. Wolff AC, Hammond ME, Schwartz JN, et al; for American Society of Clinical Oncology, College of American Pathologists. American Society of Clinical Oncology/College of American Pathologists guideline recommendations for human epidermal growth factor receptor 2 testing in breast cancer. J Clin Oncol. 2007;25(1):118-145.
22. WHO Expert Consultation. Appropriate body-mass index for Asian populations and its implications for policy and intervention strategies. Lancet. 2004;363(9403):157-163.

23. Goldhirsch A, Wood WC, Coates AS, Gelber RD, Thürlimann B, Senn HJ; for Panel members. Strategies for subtypes - dealing with the diversity of breast cancer: highlights of the St Gallen International Expert Consensus on the Primary Therapy of Early Breast Cancer 2011. Ann Oncol. 2011;22(8):1736-1747.

24. Begg CB, Zhang ZF. Statistical analysis of molecular epidemiology studies employing case-series. Cancer Epidemiol Biomarkers Prev. 1994;3(2):173-175.

25. Emaus A, Veierød MB, Tretli S, et al. Metabolic profile, physical activity, and mortality in breast cancer patients. Breast Cancer Res Treat. 2010;121(3):651-660.

26. Berstad P, Coates RJ, Bernstein L, et al. A case-control study of body mass index and breast cancer risk in white and African-American women. Cancer Epidemiol Biomarkers Prev. 2010;19(6):1532-1544.

27. Caan BJ, Kwan ML, Hartzell G, et al. Pre-diagnosis body mass index, post-diagnosis weight change, and prognosis among women with early stage breast cancer. Cancer Causes Control. 2008;19(10): 1319-1328.

28. Carey LA, Perou CM, Livasy CA, et al. Race, breast cancer subtypes, and survival in the Carolina Breast Cancer Study. JAMA. 2006; 295(21):2492-2502

29. Yang XR, Sherman ME, Rimm DL, et al. Differences in risk factors for breast cancer molecular subtypes in a population-based study. Cancer Epidemiol Biomarkers Prev. 2007;16(3):439-443.

30. Su Y, Zheng Y, Zheng W, et al. Distinct distribution and prognostic significance of molecular subtypes of breast cancer in Chinese women: a population-based cohort study. BMC Cancer. 2011;11:292.

31. Xing P, Li J, Jin F. A case-control study of reproductive factors associated with subtypes of breast cancer in Northeast China. Med Oncol. 2010;27(3):926-931.

32. Voduc KD, Cheang MC, Tyldesley S, Gelmon K, Nielsen TO, Kennecke H. Breast cancer subtypes and the risk of local and regional relapse. J Clin Oncol. 2010;28(10):1684-1691.
Therapeutics and Clinical Risk Management

\section{Publish your work in this journal}

Therapeutics and Clinical Risk Management is an international, peerreviewed journal of clinical therapeutics and risk management, focusing on concise rapid reporting of clinical studies in all therapeutic areas, outcomes, safety, and programs for the effective, safe, and sustained use of medicines. This journal is indexed on PubMed Central, CAS,

\section{Dovepress}

EMBase, Scopus and the Elsevier Bibliographic databases. The manuscript management system is completely online and includes a very quick and fair peer-review system, which is all easy to use. Visit http://www.dovepress.com/testimonials.php to read real quotes from published authors. 